\title{
Análise Quantitativa do Solvente em Cristais
}

\author{
Hamilton B. Napolitano, Solemar S. Oliveira, Ademir J. Camargo, \\ Stefano Trapani \& Glaucius Oliva
}

Um importante passo na determinação de estrutura cristalina de proteínas é a estimativa do conteúdo de solvente cristalino. O cálculo do conteúdo de solvente cristalino é normalmente baseado na estimativa do Volume de Matthews $\left(V_{M}\right)$. A estimativa do $V_{M}$ nem sempre conduz a uma única escolha para o número de moléculas de proteínas na cela unitária, em especial quando esse número é elevado. Neste trabalho apresentamos uma avaliação teórica - baseado no teorema de Parseval para a transformada de Fourier e a distribuição estatística de Wilson - da relação entre a soma de todas as intensidades experimentais difratadas e o número de átomos ordenados encontrados na cela unitária.

Palavras-chave: cristalografia de proteínas; estrutura cristalina; conteúdo de solvente.

An important step in protein crystal structure determination is the estimation of the crystal solvent content. The calculation of the crystal solvent content is commonly based on the estimation of the Matthews volume $\left(V_{M}\right)$. The estimation of $V_{M}$ does not always lead to a unique choice for the number of protein molecules, particularly when this number is high. In this work we establish a simple theoretical background — based on the Parseval theorem on Fourier transforms and the Wilson intensity statistics whereby a relationship between the sum of all experimental diffraction intensities and the number of ordered atoms in the unit cell is found.

Keywords: protein crystallography; crystal structure; solvent content. 


\section{Introdução}

Informações sobre a estrutura tridimensional de uma molécula podem ser obtidas por várias metodologias ${ }^{1}$ : difração de raios X por monocristais, difração de nêutrons por monocristais, ressonância magnética nuclear, espalhamento de raios $\mathrm{X}$ a baixo ângulo [SAXS] em solução, microscopia eletrônica, modelagem teórica, entre outros. Dentre essas, o método cristalográfico apresentase como o mais adequado devido à alta resolução com que se pode descrever a densidade eletrônica. ${ }^{1,2} \mathrm{O}$ método cristalográfico tem contribuições relevantes em diversas áreas, dentre as quais pode-se citar Genômica, Proteômica e Bioinformática. Os avanços dessas áreas têm contribuído decisivamente na identificação de alvos moleculares, abrindo novas perspectivas que vêm revolucionando as pesquisas por novos fármacos.

Uma vez identificado um alvo biológico promissor envolvido em um estado de doença humana, o processo de descoberta de novas moléculas bioativas com potencial terapêutico poderá ser iniciado. Neste momento, se dará a decisão por parte da companhia farmacêutica sobre o investimento de milhões de dólares neste longo processo, que passará, impreterivelmente, pelas fases complexas de descoberta e desenvolvimento. A abordagem moderna para o desenho racional de drogas $^{3}$ fundamenta-se no princípio da interação fármaco-receptor, que se dá através de mecanismos de complementaridade estérica, química e geométrica entre o ligante e a macromolécula alvo. Uma vez conhecida a estrutura tridimensional de uma enzima como potencial alvo patogênico, estas informações se tornam valiosas na busca de possíveis inibidores e potenciais fármacos.

O trabalho de determinação da estrutura tridimensional através da cristalografia de proteínas, como parte da pesquisa por novos fármacos, envolve as seguintes etapas ${ }^{1,2}$ : purificação da proteína, cristalização, coleta de dados, processamento dos dados, resolução da estrutura, refinamento, validação e análise do modelo cristalográfico, e ainda depósito da estrutura no banco de dados apropriado. Essa última etapa é, às vezes, suprimida quando há algum valor econômico agregado à estrutura resolvida. $\mathrm{O}$ método difratométrico fornece o conhecimento tridimensional de estruturas moleculares - a nível atômico - para compostos no estado cristalino, proporcionando confiança nos resultados em pesquisas erigidas a partir do modelo estrutural obtido.

\section{Fundamentação Teórica}

\section{ANÁLISE ESTATÍSTICA DAS INTENSIDADES E DO NÚMERO DE MATTHEWS}

Um dos primeiros passos no trabalho de determinação da estrutura cristalográfica de uma molécula protéica é a análise do conteúdo do solvente existente no cristal $^{4,5}$. Essa informação é necessária, por exemplo, na estratégia escolhida para faseamento por Substituição Molecular ou no procedimento de solvent flattering. O cálculo dessa fração do cristal ocupada por solvente é usualmente baseado no volume de Matthews $\left[V_{M}\right]$ que, em seguida, é comparada à distribuição estatística experimental, obtida primeiramente por B. W. Matthews ${ }^{7}$, em seu trabalho clássico.

A Figura 1 apresenta o gráfico obtido por Matthews para 116 estruturas cristalinas de proteínas ilustrando a largura da distribuição. Ele observou que os coeficientes angulares das retas $V_{M}$, que incluíam todos os dados analisados da Figura 1, eram 1,68 e 3,53 $\AA^{3} /$ Da para os limites inferior e superior, respectivamente. Esses valores para o $V_{M}$ correspondem às frações 27 e $78 \%$ do cristal ocupado por solvente (sendo $43 \%$ o valor mais comum).

Kantardjieff \& Rupp ${ }^{8}$ fizeram uma análise mais atual da distribuição para o $V_{M}$ baseada num conjunto de 10.471 estruturas disponíveis no Protein Data Bank [PDB $]^{9}$. Essa freqüência da distribuição do $V_{M}$ obtida é comparada com aquela obtida por Matthews em 19687 ${ }^{7}$, como pode ser visto na Figura 2. O intervalo da distribuição é ampliado e o valor de $V_{M}$ mais provável corresponde a $47 \%$ do volume do cristal ocupado por solvente. Kantardjieff \& Rupp ${ }^{8}$ observaram ainda que os cristais que difratavam a alta resolução possuíam, em geral, pequena fração do seu volume ocupado por solvente, indicando que cristais com elevado grau de empacotamento difratam melhor do que aqueles com baixo empacotamento. A resolução é assim implementada como uma informação adicional objetivando maior precisão no cálculo do $V_{M}$, de forma a diminuir ambigüidades para a fração do cristal ocupada por solvente.

Andersson \& Hovmöller ${ }^{10}$ apresentaram uma análise 


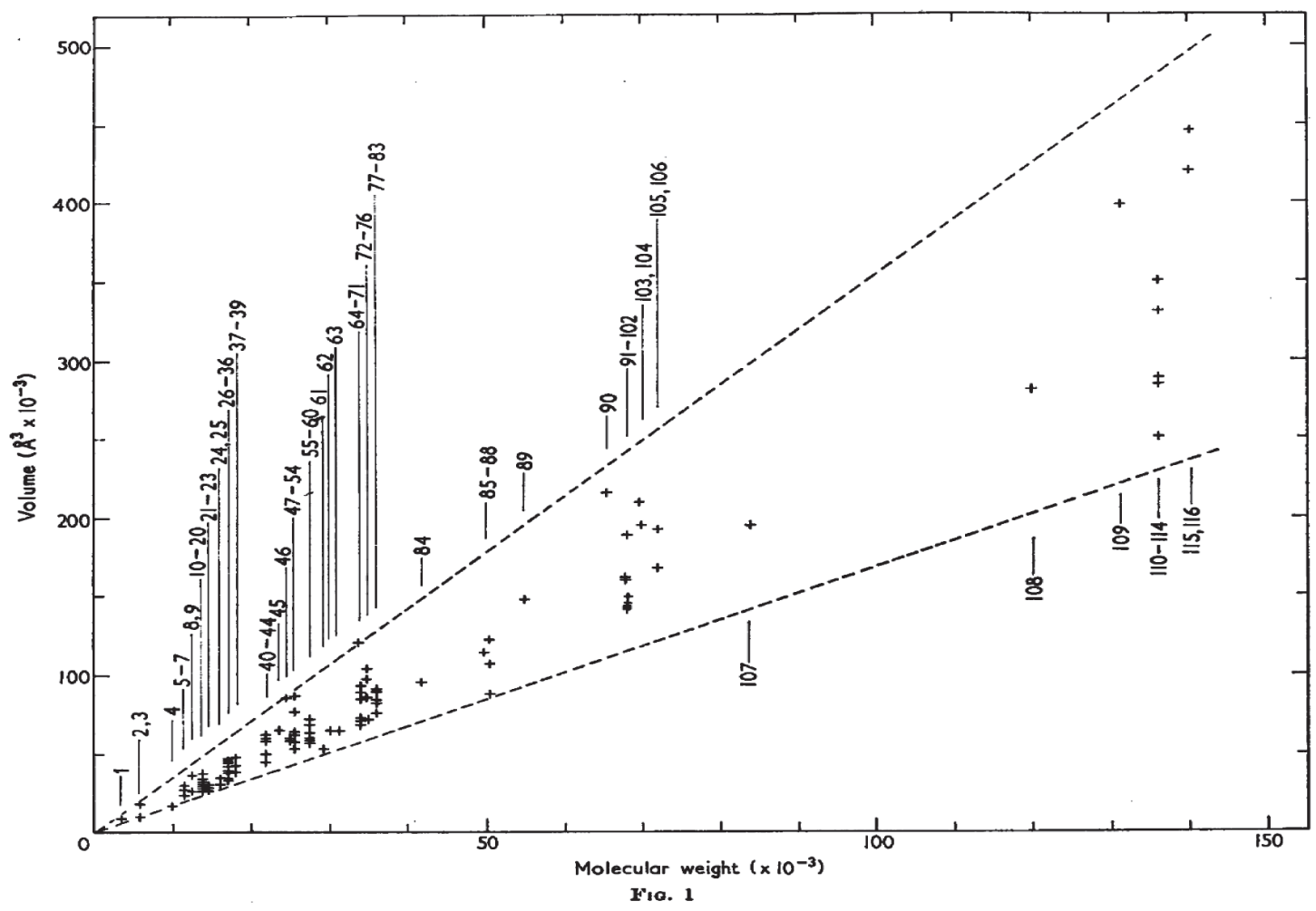

Figura 1: Gráfico ilustrando a correlação entre peso molecular e volume da cela unitária para um conjunto de 116 proteínas. Figura adaptada de [7].

estatística alternativa para o conteúdo de solvente, explorando teoricamente a correlação entre o intervalo esperado para $V_{M}$ e o grupo espacial. Assim, grupos espaciais com alto coeficiente de empacotamento ${ }^{11}$, como $P 22_{1} 2_{1} 2_{1}$ ou $\mathrm{C} 2$, devem apresentar uma largura de distribuição mais estreita do que as esperadas para grupos com baixo índice de empacotamento, como P222 ou P422. Os resultados obtidos sugerem portanto uma dependência do intervalo esperado para $V_{M}$ em relação ao grupo espacial. Contudo, o valor utilizado para a densidade volumétrica média $\left(1,22 \mathrm{~g} / \mathrm{cm}^{3}\right)$ foi fortemente combatido por Quillin \& Matthews ${ }^{12}$, argumentando a favor de um valor mais próximo do valor clássico $(1,37$ $\left.\mathrm{g} / \mathrm{cm}^{3}\right)$.

Ambos trabalhos [Andersson \& Hovmöller; Kantardjieff \& Rupp $]^{10,8}$ objetivam facilitar o cálculo da fração de solvente presente nos cristais protéicos, através do estreitamento do intervalo de $V_{M}$ em suas distribuições estatísticas. Todavia, em muitos casos, sobretudo naqueles envolvendo macromoléculas multi-oligoméricas, permanece a ambigüidade na análise do conteúdo de solvente. Nesse contexto, uma análise teórica do trabalho original de Matthews $^{7}$ abre a possibilidade para a descrição de um método alternativo de determinação da fração de solvente, presente em cristais de proteínas, a partir da análise das intensidades difratadas ${ }^{13}$.

O fator de estrutura corresponde à soma das ondas difratadas por uma cela unitária do cristal para uma dada direção. Expressa a soma do poder de espalhamento de todos os átomos da cela unitária em relação a um plano $h k l^{14}$. Está relacionada, portanto, à distribuição dos átomos da cela unitária e, a partir do conhecimento da sua distribuição eletrônica, pode-se calcular os fatores de estrutura correspondentes. A função $F(\mathbf{S})$ é não nula nos nodos do retículo cristalino recíproco ${ }^{11,14}$, sendo a transformada de Fourier da densidade eletrônica $\rho(\boldsymbol{r})$. 


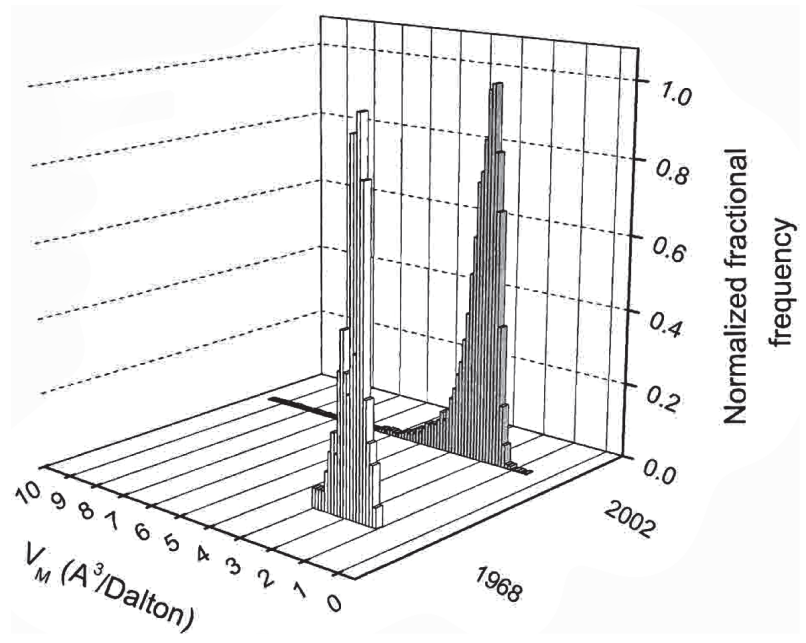

Figura 2: Gráfico tri-dimensional comparando a distribuição estatística do $V_{M}$ para 1968 e 2002. Figura adaptada de [8].

A intensidade de cada reflexão é sempre medida em escala arbitrária, e depende de vários fatores, como' ${ }^{1}$ : volume da cela, volume do cristal, tempo de exposição ao feixe dos raios $\mathrm{X}$, distância do cristal em relação ao detector, corrente e tensão do gerador do difratômetro. A formulação especial dos fatores de estrutura, denominada fator de estrutura normalizado, normaliza esta escala arbitrária das intensidades. É definido de forma que o valor quadrático médio seja igual à unidade, qualquer que seja o conjunto de intensidades coletadas (para os vetores recíprocos $\mathbf{S}$ ), sobre as quais a média é calculada. Sua definição é dada por ${ }^{1,11}$

$$
E(\mathbf{S})=\frac{F(\mathbf{S})}{\left(\varepsilon \sum_{j=1}^{N} f_{j}^{2}\right)^{\frac{1}{2}}}
$$

onde $\varepsilon$ é um número inteiro que leva em conta o efeito da simetria do grupo espacial sobre as intensidades, e a quantidade entre parênteses no denominador é a intensidade média esperada. Enquanto os fatores de estrutura 'medidos experimentalmente' correspondem a $N$ átomos distintos com dimensão finita que vibram termicamente, os fatores de estrutura normalizados correspondem a um modelo com $N$ átomos iguais, puntiformes e sem vibração térmica ${ }^{15,16}$, além de não levarem em conta o ângulo de espalhamento $\theta$. A Figura 3a apresenta o comportamento do fator de espalhamento atômico e o comportamento do átomo puntiforme em repouso. Figura 3b.

O valor médio da distribuição dos fatores de estrutura normalizados é dado por ${ }^{1,11}$

$$
\left\langle|E(\mathbf{S})|^{2}\right\rangle=\frac{\sum|E(\mathbf{S})|^{2}}{N_{\text {ref }}}=1
$$

onde $N_{\text {ref }}$ é o número total de reflexões. Verifica-se a partir da Equação (2) que a somatória sobre o número total de reflexões (numerador da Equação 2) é igual a $N_{r e f}$. Como a quantidade total de reflexões $N_{\text {ref }}$ é diretamente proporcional ao volume da cela unitária Ladd \& Palmer (1994) $)^{17}$, o trabalho de Matthews (1968) corresponde a uma análise da distribuição estatística dos fatores de estrutura normalizados avaliados versus o peso molecular das proteínas. Essa identificação nos motivou a buscar outras formas de distribuições estatísticas que fossem úteis na análise do conteúdo de solvente.

\section{AVALIAÇÃO DO CONTEÚDO DE SOLVENTE}

O cálculo do $V_{M}$ exige a priori o conhecimento do peso molecular da proteína e do volume da cela unitária ${ }^{1,7}$. Adicionalmente, o número de proteínas contidas na cela unitária é uma informação necessária. Nem sempre o resultado obtido leva a uma única escolha do número de moléculas na cela, principalmente quando este número é relativamente grande. Isso ocorre devido à largura da distribuição de Matthews ${ }^{7}$, conforme apresentado na Figura 1, que, às vezes, leva a ambigüidades no número de unidades protéicas, especialmente se a proteína apresenta estrutura quaternária multimonomérica.

Uma forma alternativa, baseada no teorema de Parseval para a transformada de Fourier $^{18}$ e na análise estatística das intensidades feita por Wilson ${ }^{14,19}$, é desenvolvida, objetivando estimar o conteúdo de solvente de um dado cristal. Wilson mostrou ${ }^{19}$ que a intensidade média de uma reflexão geral é $\langle I(\mathbf{S})\rangle=\sum_{j=1}^{N} f_{j}^{2}$. Partindo 


\section{Artigo 4}

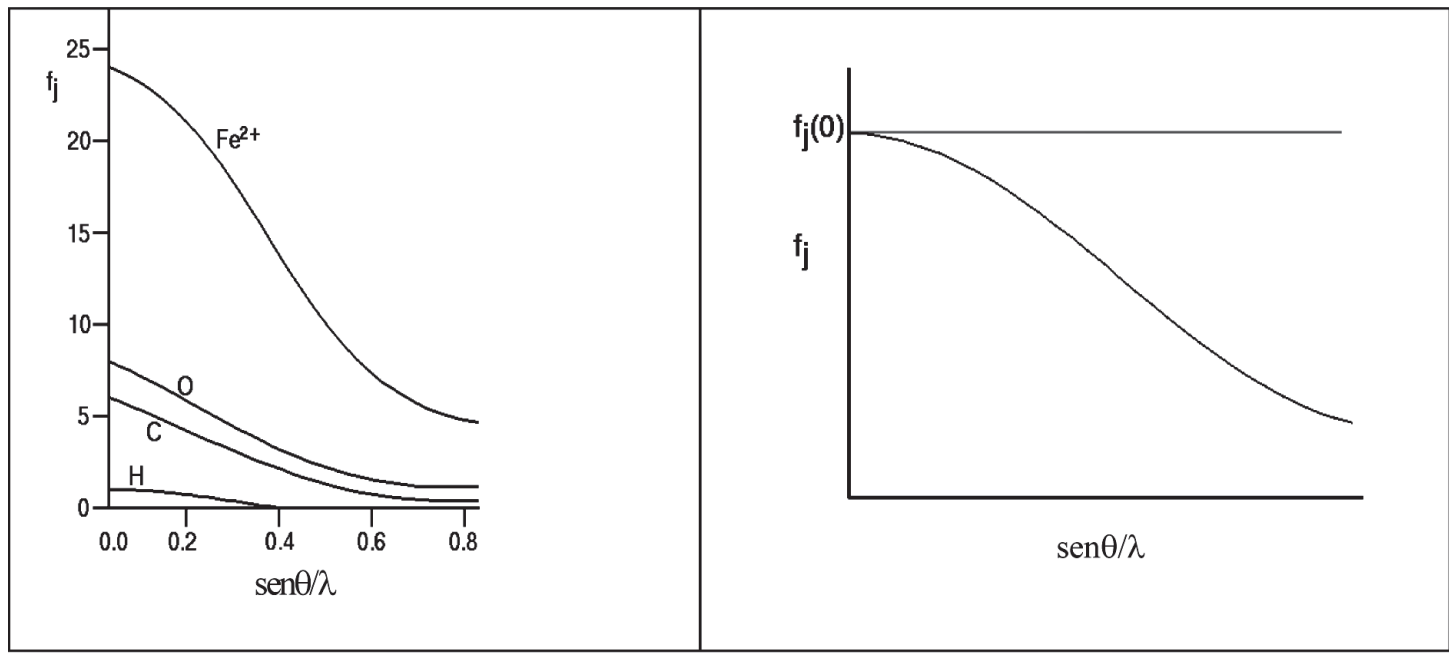

(a)

(b)

Figura 3: Dependência do fator de espalhamento atômico $f$ em relação a sen $\theta / \lambda . f_{\mathrm{j}}(0)=\mathrm{Z}$ corresponde ao número de elétrons do átomo. (a) Ilustração quantitativa do fator de espalhamento atômico para alguns átomos típicos em estruturas cristalinas; (b) Comportamento qualitativo para um átomo qualquer; a reta horizontal refere-se a um átomo hipotético pontual e estacionário.

desse resultado, ele analisou a distribuição estatística das intensidades chegando à expressão

$\ln \frac{\left\langle I_{\text {exp }}\right\rangle}{\sum_{j}\left(f_{j}^{O}\right)}=\ln \left[K \exp \left(\frac{-2 B \operatorname{sen}^{2} \theta}{\lambda^{2}}\right)\right]=\ln K-2 B \frac{\operatorname{sen}^{2} \theta}{\lambda^{2}}$

onde $f_{\mathrm{j}}^{0}$ corresponde ao fator de espalhamento do átomo em repouso, $\lambda$ ao comprimento de onda da radiação $\mathrm{X}, B$ ao parâmetro de deslocamento atômico ${ }^{20}$ e $K$ à constante arbitrária que leva as intensidades coletadas $I_{\text {exp }}(\mathrm{S})$ à escala absoluta, devendo satisfazer a relação $\left\langle I_{\exp }(\mathbf{S})\right\rangle=K\left\langle I_{a b s}(\mathbf{S})\right\rangle$.

A Equação (3) contém três variáveis desconhecidas: $K, B$ e $\sum_{j}\left(f_{j}\right)^{2}$ de forma que a utilidade prática de levar o conjunto coletado da escala arbitrária para escala absoluta fica limitada ao conhecimento, a priori, da quantidade de elétrons dentro da cela unitária. Dada a existência do solvente nos cristais de proteínas que, conforme citado anteriormente, ocupam entre 20 e $80 \%$ do volume, esta avaliação se torna prejudicada.

Representando cada átomo protéico por um átomo hipotético de número atômico médio igual a $Z_{M}$, obtém-se $\sum_{j}\left(f_{j}^{o}\right)^{2}=N_{a t}\left(Z_{M} \bar{f}^{0}\right)^{2}$ onde $\bar{f}^{0}$ corresponde à curva padrão do espalhamento atômico ${ }^{11,14}$. Uma hipótese similar éamplamenteutilizada,com sucesso, nateoriados Métodos Diretos $^{21,22}$, para a solução de estruturas cristalográficas de moléculas pequenas. Esta sugestão surge da constatação que as moléculas de proteínas são constituídas por átomos de composição atômica pouco variável.

Assim pode-se re-escrever o gráfico de Wilson ${ }^{1}$, Equação (3), da seguinte forma

$$
\ln \frac{\left\langle I_{\exp }\right\rangle}{\left(Z_{M} \bar{f}^{0}\right)^{2}}=\ln \left(K N_{a t}\right)-2 B \frac{\operatorname{sen}^{2} \theta}{\lambda^{2}}
$$


A Equação (4) contém somente duas incógnitas, $K N_{\text {at }}$ e $B$, tendo em vista que, em geral, macromoléculas possuem um único valor de $Z_{M}$ e que proteínas possuem a mesma densidade eletrônica ${ }^{12}$. O valor de $Z_{M}$ pode assim ser obtido por alguma análise estatística do conjunto de estruturas já depositadas em banco de dados como o $\mathrm{PDB}^{9}$. A quantidade $K N_{a t}$ guarda conjuntamente as informações do fator de escala $K$ e da quantidade de átomos ordenados dentro da cela unitária, e pode ser obtida a partir da aplicação experimental do teorema de Parseval, demonstrado por $^{18}$

$$
\int_{-\infty}^{\infty}|F(\mathbf{S})|^{2} d \mathbf{S}=\int_{\text {cela }}|\rho(\mathbf{r})|^{2} d \mathbf{r}
$$

A Equação (5) diz que a soma das intensidades difratadas (em escala absoluta) sobre todo o espaço recíproco é numericamente igual à integral da densidade eletrônica sobre toda a cela unitária. A idéia central é que a quantidade total de energia, difratada por uma cela, está diretamente relacionada com a quantidade de átomos espalhadores presentes na cela unitária. O primeiro termo (somatório sobre todas as intensidades difratadas para o estado cristalino) e o segundo (integral da densidade eletrônica elevada ao quadrado) da Equação (5) são respectivamente iguais a

$$
\begin{gathered}
\int_{-\infty}^{\infty}|F(\mathbf{S})|^{2} d \mathbf{S} \approx \sum_{\mathbf{S} \neq 0} I_{a b s}+I_{a b s}(000)=\sum_{\mathbf{S} \neq 0} I_{a b s}+\left(N_{a t} Z_{M}\right)^{2} \\
\int_{\text {cela }}|\rho(\mathbf{r})|^{2} d \mathbf{r} \approx N Z_{M}\left\langle\int \rho_{M}^{2}\left(\mathbf{r}-\mathbf{r}_{M}\right) d \mathbf{r}\right\rangle=N_{a t} Z_{M} \xi
\end{gathered}
$$

onde $\rho_{M}^{2}\left(\mathbf{r}-\mathbf{r}_{M}\right)$ é o quadrado da densidade eletrônica média no domínio da cela unitária, e $\xi$ é o valor médio da integral do quadrado da densidade eletrônica, para o átomo hipotético com número atômico $Z_{M}$, sendo igual a $\xi \equiv\left\langle\int \rho_{a t}^{2}(\mathbf{r}) d \mathbf{r}\right\rangle$. Aplicando as duas equações acima na Equação (5) chega-se à relação aproximada

$$
\sum_{\mathbf{S} \neq 0}\left(\frac{I_{\exp }}{K V N_{a t}}\right) \approx \xi Z_{M}-\frac{N}{V}\left(Z_{M}\right)^{2}
$$

onde $V$ é o volume da cela unitária. A viabilidade prática e genérica da Equação (8) precisa levar em conta as contribuições relevantes como o parâmetro de deslocamento atômico $B$ (particular de cada cristal) e o espalhamento advindo do solvente desordenado. Adicionalmente, parâmetros como completeza, resolução e presença de átomos pesados, dentre outros, precisam ser considerados. Contudo, a Equação (8) mostra que existe uma relação útil entre a soma das intensidades difratadas (em escala arbitrária) e o conteúdo eletrônico ordenado. A quantidade KNat para uma estrutura desconhecida pode ser obtida a partir da Equação (4), fazendo uso somente do conjunto de dados reduzidos. $\mathrm{O}$ valor médio $Z_{M}$ pode ser obtido através do coeficiente angular dessa curva.

O gráfico da Figura 4 ilustra a consistência da previsão teórica da Equação (8) para o conjunto das 213 estruturas cristalográficas, extraídas do PDB, apresentado na Tabela 1. A seleção dessas estruturas foi realizada através do mecanismo de busca do PDB, de forma a descartar estruturas de carboidratos, ácidos nucléicos e seus complexos com proteínas (retendo apenas estruturas de proteínas), e a descartar ainda proteínas que apresentassem um grau de homologia maior que $90 \% \mathrm{com}$ outro ítem do mesmo grupo, evitando a presença de ítens redundantes no conjunto final. Entre os ítens homólogos, o critério de escolha foi baseado na resolução máxima dos correspondentes dados de difração. O peso molecular do conteúdo da cela variou entre 4,6 e $306 \mathrm{KDa}$.

Os dados apresentados na Figura 4 correspondem aos fatores de estrutura calculados em vez dos observados, obtidos através do programa SFALL do pacote de programas Collaborative Computational Project Number $4[\mathrm{CCP} 4]^{23}$, evitando assim ajustes com a resolução, a completeza, a presença de átomo pesado e a contribuição do solvente desordenado, entre outras.

A correlação entre as intensidades espalhadas e o conteúdo eletrônico espalhador dá as diretrizes para um possível método alternativo na análise do conteúdo de 


\section{Artigo 4}

Tabela 1: Lista dos códigos relativos às 213 estruturas escolhidas no PDB para a análise da Equação (8).

1a2p 1byz 1e9w 1 fw9 1hbz 1jks 1kth 1mn8 2ae2 1gk8 1jec 1a3g 1bzp 1eaq 1fxo 1hfx 1jpu 1ktz 1n40 2tps 1gkp 1n21 1a69 1bzy 1eay 1g2p 1hg7 1jpz 1kw3 1naw 2ucz 1jz8 1qhx 1a6m 1c0p 1eb8 1g6x 1hix 1jr0 1kwn 1ney 2ush 1ko7 2occ 1a7y 1c4x 1ed9 1g7a 1hiz 1jsf 1ky3 1nks 3al1 1moq 1be3 1a7z 1c5u 1ejg 1g7b 1hj6 1jvb 1kyc 1 o61 31zt 1obr 1ck7 1a8u 1c75 1ek6 1ga6 1hqs 1jxu 113f 1o7j 3pyp 1ryp 1dwv 1a9y 1cjc 1en2 1gjn 1i40 1k05 116r 1pig 3std 2xat 1f2j 1aqm 1cru 1ewy 1gkz 1i4u 1k2a 1171 1qg0 4lzt 1az9 4csm lavz 1ctq 1f4b 1gnx 1i6w 1k3i 1191 1qi9 4pga 1b7y 1 f49 1b0y 1dgf 1f98 1go3 1i8o 1k6u 1leh 1qip 4ubp 1bcc 1k47 $1 \mathrm{~b} 12$ 1dhp 1fd3 1gq6 1ie7 1kb0 1lni 1qje 5eau 1ft1 1qo5 1b6r 1djr 1fdy 1gtv 1inl 1kf3 11s9 1qke 1a9x 1gxd 1jky 1be4 1duc 1fhe 1gtz 1iua 1kfc 1lu0 1qow 1avq 1h5q 1bez 1dvj 1fj2 1gwc 1j16 1kgs 1lz8 1qq5 1b3o 1jaw 1bgy 1dxe 1flm 1gyo 1j8q 1kjq 1m1q 1qqf 1bj4 1k7h 1 bl5 1dxy 1fm8 1gzu 1j9b 1kmv 1m24 1rdr 1civ 1pys 1bn5 1dy5 1fo8 1h05 1jg1 1koi 1m40 1shk 1cp9 1tr1 1bn7 1e4c 1fsf 1h2j 1jh6 1kp4 1m7g 1ush 1e1o 3bta 1brt 1e93 1fsg 1h6r 1jig 1ks8 1ml7 1vns 1e4i 1ftr

solvente para cristais de proteína. Quando essa correlação é aplicada para a interação dos raios $\mathrm{X}$ com a matéria no estado amorfo, ou seja, é aplicada ao espalhamento a baixos ângulos [SAXS], a informação obtida será o peso molecular da unidade biológica monodispersa em solução ${ }^{24,25,26}$.

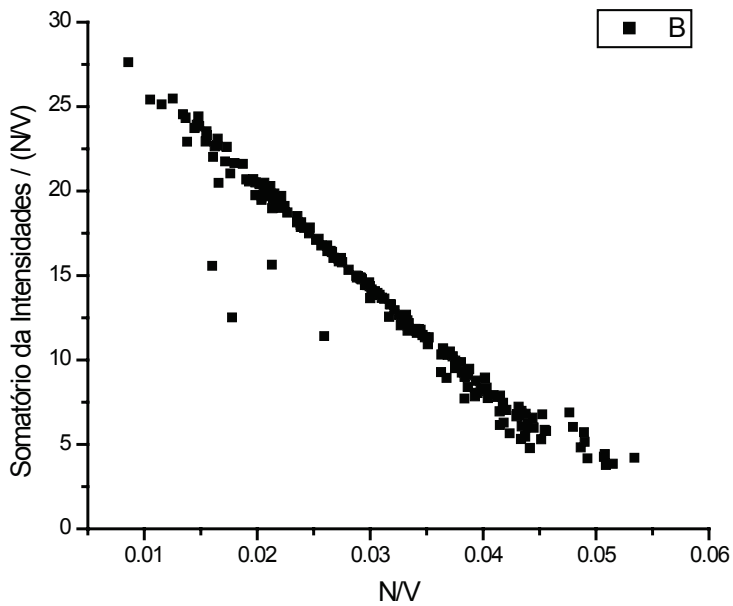

Figura 4: Gráfico ilustrando a correlação entre a somatória das intensidades observadas e o conteúdo eletrônico da cela unitária.

\section{Discussão dos resultados}

Considerando que a resolução da estrutura é uma etapa fundamental no trabalho de determinação da estrutura tridimensional através da cristalografia de proteínas, a Equação (8) torna-se uma alternativa para a análise do conteúdo de solvente (presentes em cristais de macromoléculas), podendo acelerar o processo de determinação da estrutura cristalográfica de tais receptores biológicos, uma vez que o conhecimento do conteúdo de solvente é um importante passo na obtenção da resolução da estrutura cristalográfica.

\section{REFERÊNCIAS}

1. Drenth, J. Principles of Protein Xray

Crystallography. 2nd ed., Springer, New York. 1999.

2. Giacovazzo, C.; Monaco, H.L.; Viterbo, D.; Scordari, F.; Gilli, G.; Zanotti, G. \& Catti, M. Fundamentals of Crystallography. 2nd ed., IUCr e Oxford Science Publication, New York. 2002. 
3. Blundell, T.L. Structure $\neg$ based drug design. Nature 384, Supplement. 1996. 23.

4. McPherson, A. Introduction to Macromolecular Crystallography. John Wiley, New Jersey. 2003.

5. Carter, C. W. \& Sweet, R. M. Methods in Enzymology, Vol. 276. Macromolecular Crystallography Part A. ed. Academic Press, San Diego, 326. 1997.

6. Carter, C.W. \& Xiang, S. Phase Improvement Using Conditional Probability Methods: Maximum Entropy Solvent Flattening and Phase Permutation. In Methods in Enzymology, Vol. 277. Macromolecular Crystallography Part B. C.W. Carter \& R.M. Sweet ed. Academic Press, San Diego, 79. 1997.

7. Matthews, B. W. Solvent Content of Protein Crystals. J. Mol. Biol. 1968. 33, 491.

8. Kantardjieff, K. A. \& Rupp, B. Matthews coefficient probabilities: improved estimates for unit cell contents of proteins, DNA, and protein-nucleic acid complex crystals. Protein Science. 2003. 12, 1865.

9. Berman, H. M.; Westbrook, J.; Feng, Z.; Gilliland, G.; Bhat, T. N.; Weissig, H.; Shindyalov, I. N. \& Bourne, P. E. The Protein Data Bank. Nucleic Acids Research. 2000. 28 pp. 235.

10. Andersson, K. M. \& Hovmöller, S. The protein content in crystals and packing coefficients in different space groups. Acta Cryst. 1999. D56, 789.

11. Giacovazzo, C. The Diffraction of X-ray by Crystals. In: Fundamentals of Crystallography, 2nd ed. Editado por Giacovazzo, C. IUCr e Oxford Science Publication, New York. 2002.

12. Quillin, M. \& Matthews, B. W. Accurate calculation of the density of proteins. Acta Cryst. 2000. D56, 791.
13. Napolitano, H. B.; Trapani, S.; Fischer, H.; Craievich, A. F. \& Oliva, G. Is it possible to determine the solvent content of the protein crystals from diffraction intensities analysis? In:

Livro de Resumos da XVI SBCr. Editado por Napolitano, H. B. \& Mascarenhas, Y. P. SBCr, São Carlos, 17. 2003.

14. Stout, G. \& Jensen, L. H. X-ray Structure Determination - a practical guide, 2nd ed. The Macmillan Company. London. 1989.

15. Cullity, B. D. Elements of X-ray Diffraction, nd ed. Addison-Wesley Publishing Company, Inc. Massachusetts. 1978.

16. Schwarzenbach, D. Crystallography. John Wiley e Sons. Chichester. 1996.

17. Ladd, M. F. C. \& Palmer, R. A. Structure Determination by X-Ray Crystallography, 2nd Ed. Kluwer Academic Pub, London. 1994.

18. Brigham, E. O. The Fast Fourier Transform. Englewood Cliff, New Jersey. 1974.

19. Wilson, A. J. C. The probability distributions of $X$ ray intensities. Acta Crystallogr. 1950. 3, 397.

20. Castellano, E. E. \& Main, P. On the classical interpretation of thermal probability ellipsoids and the Debye-Waller factor. Acta Cryst. 1985. A41, 156.

21. Napolitano, H. B. Determinação por Difração de Raios-X das Estruturas Cristalinas de Enaminonas e N-Benzoil-Guanidina com Potencial Atividade Anticonvulsivante. Dissertação de mestrado. Instituto de Física, UFG. 2001.

22. Viterbo, D. Solution and Refinement of Crystal Structure. In: Fundamentals of Crystallography, 2nd ed. Editado por Giacovazzo, C. IUCr e Oxford Science Publication, New York. 2002. 
23. CCP4. The CCP4 Suite: Programs for Protein Crystallography. Acta Cryst. 1994. D50, 760.

24. Fischer, H.; Napolitano, H. B.; Oliva, G. \& Craievich, A. F. Protein molecular weight determination on an arbitrary scale by SAXS. Journal of Applied Crystallography. Artigo em preparação. 2004.

25. Napolitano, H. B.; Oliva, G.; Fischer, H.; Craievich, A. F. Molecular weight determination on an arbitrary scale by SAXS. In: ACA 2003 Annual Meeting: program and abstract book. SBCr, Covington. 2003.

26. Branden, C. \& Tooze, J. Introduction to Protein Structure. Garland Publishing, New York. 1991.

\section{Hamilton B. Napolitano*1, Solemar S. Oliveira', Ademir J. Camargo', Stefano Trapani $^{2}$ \& Glaucius Oliva ${ }^{3}$}

\author{
${ }^{1}$ Ciências Exatas e Tecnológicas, UEG. PO Box 459, Anápolis, GO, \\ 75001-970, Brazil \\ ${ }^{2}$ Centre de Biochimie Structurale (CBS). 34090 MONTPELLIER. \\ France. \\ ${ }^{3}$ Instituto de Física de São Carlos, USP. PO Box: 369, São Carlos, SP, \\ 13566-590, Brazil. \\ *E-mail: hnapolitano@pq.cnpq.br
}

